\title{
A Tactile Enhancement Instrument for Minimally Invasive Surgery
}

\author{
Hsin-Yun Yao, Vincent Hayward \\ Haptics Laboratory \\ Center for Intelligent Machines, McGill University,Montréal, Canada, \\ Randy E. Ellis \\ School of Computing, Queen's University, Kingston, Canada \& \\ Department of Radiology, Harvard Medical School, Boston, USA
}

(A preliminary version of this paper appeared in proceedings of MICCAI 2004, pp. 89-96.)

\begin{abstract}
Objective: Surgeons use probes during during minimally invasive arthroscopy as diagnostic tools to detect tissues anomalies. Improving tactile sensitivity during this activity would be valuable.

Materials and Methods: We developed an enhanced probe that could heighten the tactile sensations experienced while probing objects. It operated by detecting the acceleration signal resulting from the interaction of the tool tip with surfaces and magnifying it for tactile and auditory reproduction. The instrument consisted of an accelerometer and an actuator arranged such that the sensing direction was orthogonal to the actuating direction so as to decouple input from output. Using the instrument, subjects were asked to detect cuts under four conditions: with no amplification, with enhanced tactile feedback, with sound feedback, and with passive touch.

Results: We found that, for tactile reproduction, the current prototype could amplify the signals by $10 \mathrm{~dB}$ on average. Results from statistical methods showed significant improvements of performance in the case of tactile and auditory feedback.

Conclusion: We developed a surgical probe with tactile and auditory feedback. Despite the moderate system gain achievable by the initial prototype, the system could measurably improve users' ability to detect small cuts in cartilage-like elastic surfaces.
\end{abstract}

Keywords: arthroscopy; haptic devices; force feedback; multi-modal interfaces; computerassisted orthopedic surgery 


\section{Objective}

Minimally invasive surgery benefits patients because of smaller incisions, less pain, less trauma and shorter healing periods, but the surgeon must cope with loss of direct tactile information and reduced visual information. It has been demonstrated that even partial restoration of touch improves performance in teleoperation and virtual environments. ${ }^{1,2,3,4}$ "Augmented reality" techniques can be used to improve human performance in surgical applications; however, systems typically have features presented graphically and so provide little in terms of haptic feedback. ${ }^{5}$

We developed an integrated instrument - termed a 'MicroTactus' - to enhance tactile sensations experienced during surgery, specifically during tissue examination with minimally invasive procedures. Our first prototype was an arthroscopic probe that measured the tip acceleration signal resulting from the interaction with an object and actively amplified it at the handle. The same signal could also be transformed into sound to heighten sensitivity to small details even further.

During diagnostic arthroscopy surgery in a joint, a surgeon inserts a small camera in one incision and a surgical probe in another. It is common for cartilage to be damaged in regions that cannot be seen from the camera, in which cases a surgeon must rely completely on haptic feedback obtained from the surgical probe. One common arthroscopic probe has a metal tip and a handle. The tips may have many different shapes, but the "arthroscopic hook" with a tip bent to a 90-degree angle is commonly used. With this instrument, a surgeon probes the surface of tissues, including ligaments, menisci and cartilage, to find anomalies.

The arthroscopic hook shown in Figure 1 has an accelerometer embedded near its tip, as well as a custom-designed actuator in the handle. The system could amplify the acceleration signal uniformly, but the probe's response varied according to gripping methods and probed surfaces making the complete system analysis quite difficult. However, a preliminary design comprising bandpass filtering and amplification resulted in a useful system that could be demonstrated to enhance human performance in a small cut detection task. The results indicated that, with even rudimentary signal processing in the haptic and auditory domains, tear-detection performance was significantly improved.

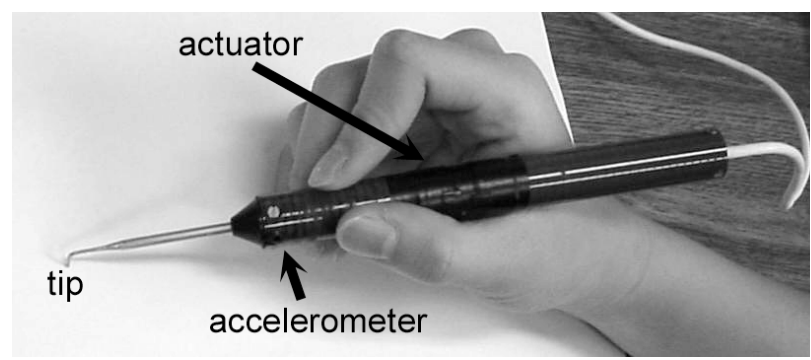

Figure 1: Application of the MicroTactus concept to an augmented arthroscopic probe. 


\section{Methods and Materials}

\subsection{Principle of Operation}

We determined which signal(s) to detect and amplify by considering the motion of a probe as it interacts with a surface. The motion depends on the external forces applied, which for a probe are the interaction contact forces and the user's grip forces. These combined forces are the forcing term of the probe's dynamics, which in turn are its rigid-body dynamics and structural dynamics. The user's tactile information is derived entirely from the deformation of the tissues of the hand holding the probe, whether the action is to press on, drag over, or tap a surface. This information is highly dependent on the dynamics of the probe and on the size and shape of the tip. Nevertheless, each of these actions, or combination thereof, informs the user of the properties of the tissues under test.

From this analysis we concluded that the sensory function of a probe is to transfer the movements of its tip to movements of the tissues at its interface with the hand. For tactileenhancement purposes, all we needed to sense and amplify was acceleration because, with appropriate integration constants, acceleration entirely describes the movement of any object: sensing force and/or strain is unnecessary. This analysis also suggested that the appropriate tactile transducer is an actuator that can accelerate the handle.

One aspect of these movements that cannot be processed is the component resulting from applying a slow-varying force to the surface. However, in a probing task, it is presumed that subjects apply specific force intensities that maximize the information acquired from the resulting tactile sensations. What our system does is record and enhance the movements produced by this force, but not the force itself. For a given intensity smaller undulations will appear larger, and thus are easier to detect. Moreover, because the acceleration signal is highly structured and spectrally rich, if it is converted into an acoustic signal then it might be usefully processed by the auditory system for multimodality interpretation.

The device could also be used as a stimulator when not in contact with a surface. For example, with two devices, we could use one hand to manipulate the probe and the other to experience the surface; alternatively, it was possible to have an assistant scratch and tap a surface while experiencing this physical interaction remotely. The device could also be used as a surface-recording tool. For example, we could record what a surgeon experienced during arthroscopy and play it back to one or several trainees for instruction. Because of its spectral characteristics, the signal could also be recorded, played back, or monitored with an ordinary audio system (e.g., the haptic signal could be recorded in the audio portion of an ordinary audiovisual recording, such as a camcorder tape, and be replayed as a visual signal with additional haptic display).

\subsection{Hardware Design}

We applied these design principles to the design of a MicroTactus-type arthroscopic probe, which we wanted to be similar in structure and use to a conventional arhtroscopic probe. As shown in Figure 2, a biocompatible metal hook was attached to a handle made from carbon fiber tubing that was $15 \mathrm{~mm}$ in diameter. An accelerometer was mounted where the 
probe's metal tip was connected to the handle. Preliminary trials indicated that scratching a soft surface produced accelerations of about $\pm 2 \mathrm{~g}$; for harder surfaces, such as wood or plastic, the scratching acceleration was about $\pm 5 \mathrm{~g}$; and knocking on a wooden surface, or scratching it at high speed, could yield up to $\pm 10 \mathrm{~g}$. A $2 \mathrm{~g}$ dual-axis accelerometer (Analog Devices, ADXL311) was selected for a tear-detection task based on these preliminary design guidelines.

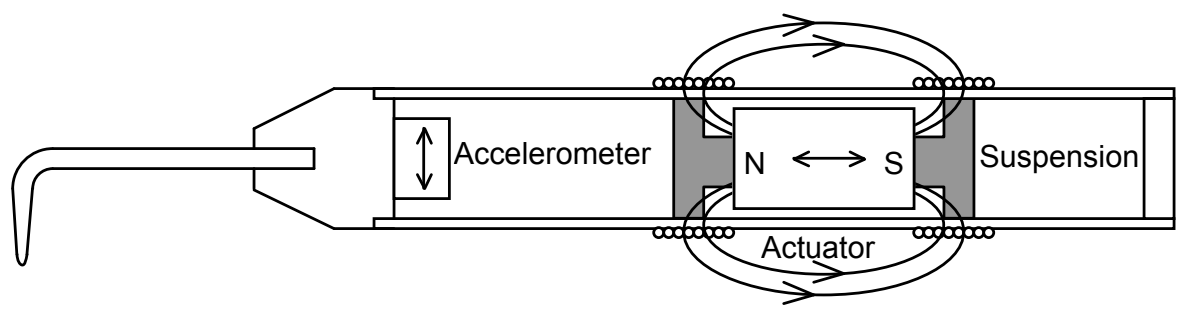

Figure 2: Main structural components of the probe. The digital signal processing hardware and software is not shown here.

After numerous iterations, we designed a structure comprising a cylindrical rare earth magnet $(\mathrm{NdFeB})$ elastically suspended inside the handle. To a good approximation the field lines were axial symmetric when escaping the magnet and crossed the loops of two coils at right angles, thereby developing a magnetic force between the magnet and the handle when current flowed. Although there are alternative designs (e.g., using variablereluctance actuators) or optimized designs (e.g., using a tubular soft-iron magnetic return), this "open magnetic circuit" design was appropriate for our immediate needs.

Maximizing acceleration of the handle's shell required minimizing its mass, maximizing the mass of the magnet, and maximizing the magnetic force. The shell was made of a carbon-fiber tube, which was light and rigid. We selected the largest magnet that could fit inside the shell and, because its weight was more than twice that of the handle, its relative mass was large. Finally, to maximize the Lorentz force between the magnet and the handle, a pair of counter-rotating coils was used to maximize the magnetic flux passing through the surface defined by the wires. We found that two layers of coiling wire gave a satisfactory result with a coil impedance similar to that of an audio transducer. A mere $10 \mathrm{~W}$ of electrical power caused vibrations to be strong enough to numb the fingers in wide range of frequencies. This low power consumption, and the modest spectral requirements, enabled us to use an ordinary audio amplifier to drive the device.

The accelerometer detected the radial components of the acceleration, whereas the actuator created axial accelerations. This had the effect of dynamically decoupling the input from the output, which was necessary because vibrations were transferred through the probe's structure. In free air, the device remained stable, even with high feedback gains.

A digital signal-processor (Analog Devices Blackfin533) was used to perform filtering and signal shaping. This processor provided us with sufficient processing power to implement digital filters and provided room for future enhancements and control algorithms. It also allowed us to record and play tactile signals. Accelerations were sampled with 16-bit resolution at $48 \mathrm{kHz}$. 


\section{Computer Aided Surgery, Vol. 10, No. 4, pp. 233-239.}

\subsection{Signal Processing}

The system could operate by directly feeding the accelerometer's signal to the actuator's amplifier. However, a filter that removed noise, anti-aliased the signal, and enhanced system stability was found to increase the usefulness of the system. The raw acceleration signal was first low-pass-filtered to remove unwanted high-frequency components above $500 \mathrm{~Hz}$. The filter had a sharp attenuation at the stopband $(50 \mathrm{~dB})$ and a flat response at the passband ( $<1 \mathrm{~dB}$ ripple).

The signal was then down-sampled from $48 \mathrm{kHz}$ to $2.4 \mathrm{kHz}$. This eased the design and implementation of filters operating on preprocessed signals below $1.2 \mathrm{kHz}$, a bandwidth sufficient for haptic signals. Many other linear and nonlinear signal-shaping filters could be used to enhanced perception in task-specific applications. In this work, however, a simple band-pass filter ( 40 to $400 \mathrm{~Hz}$ ) was found to be useful in that it improved stability when the probe was in contact with an elastic surface by eliminating the first resonant mode of the actuator. Nonetheless, many other designs to improve robustness and achieve perceptual effects are possible and have been left for future work.

\subsection{Preliminary User Study}

We carried out a preliminary experiment to evaluate the utility of the probe during the task of superficial tear detection in soft tissues. In this task, a probe was dragged over the surface to be tested. If there was a crack, the tip diped slightly, producing a transient signal that could be detected by touch. If the crack was sufficiently deep relative to the radius of the probe tip, and/or if the normal force was sufficiently high, then the tip caught the lip of the crack and thereby produces a large transient. These, and perhaps other cues, could be used by surgeons to detect and characterize surface anomalies. Typical examples of signals produced by the samples described next are shown in Figure 3. We tested the ability of subjects to detect such cracks with various uses of the MicroTactus arthroscopic probe.

Surface Preparation. In order to approximate the conditions of tear detection during arthroscopy we prepared $3 \mathrm{~mm}$-thick-pads made of Viton, a high-performance fluoroelastomer that resembles cartilage. Four $10 \times 30 \mathrm{~mm}$ pads were glue-mounted on small boxes for easy handling. Cuts were made on the surface of the pads with a sharp blade protruding by a set distance out of a block of hard rubber. One pad had no cut; the second pad had one crack $1.5 \mathrm{~mm}$ deep; the third pad had two cracks $1.5 \mathrm{~mm}$ deep; the fourth pad was completely cut to a depth of $3 \mathrm{~mm}$.

Subjects. We recruited 8 healthy individuals of age 22 to 28 . Two were physicians and six were students from the Electrical Engineering Department of McGill University. Four subjects were completely unfamiliar with our work, and the other four subjects had used the device before the experiments but did not not know the details of its design.

Procedure. Two identical MicroTactus probes were connected to the signal-processing system. Subjects sat at a table, held one probe with their dominant hand, and used 
Computer Aided Surgery, Vol. 10, No. 4, pp. 233-239.

$\mathbf{a}$
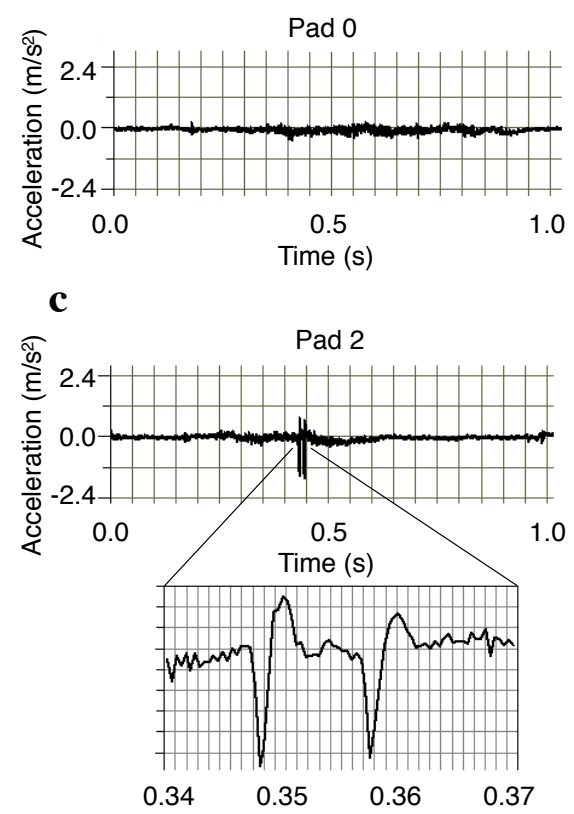

b

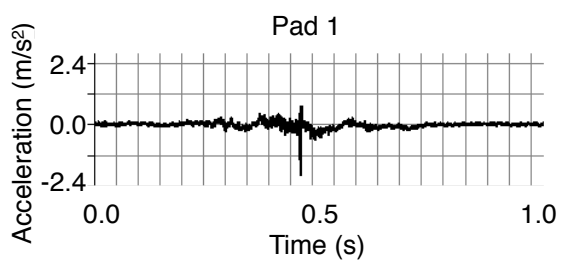

d

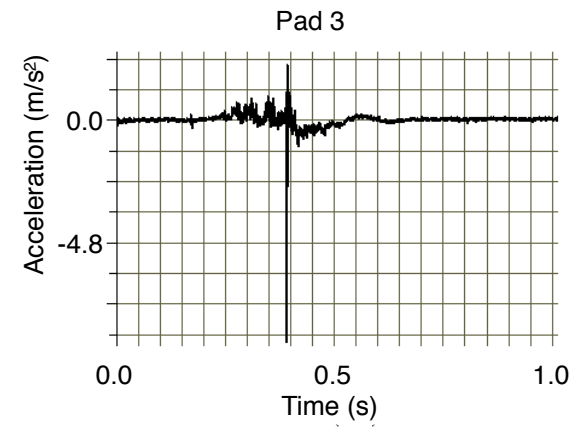

Figure 3: a) Texture amplitude was modulated by varying the pressure of the probe on the surface. b) A small crack produced a single transient. c) A double crack produced a double transient. d) A large transient given by a deep cut.

it to explore the surface of the samples while using the other hand to hold the sample mounted on the boxes. The subjects were trained in the task under the guidance of the experimenter. During the trials, the lights of the windowless room were dimmed so that it was no longer possible to see the cuts but the pads could be found on the table. A sequence of 24 pads was given to each subject in a randomized order, each pad being presented 6 times. Subjects were asked to detect if there was a cut in the pad. They had to decide rapidly and answered by pressing keys labeled YES and NO. The duration of each testing session was less than one hour. Trials were administered under four conditions in the following order:

1. Haptic: subjects explored the pads with tactile feedback activated on the same probe used for exploration.

2. Audio: subjects explored the surface with the probe, but instead of tactile feedback, audio feedback was relayed through a loudspeaker.

3. Passive: the experimenter explored the pads with a first probe, attempting to keep a constant speed. The tactile feedback from the first probe was sent to the second probe which was passively held by the subject.

4. Off: The subjects use a probe without tactile or audio feedback. 


\section{Results}

\subsection{Device Characterization}

A system analyzer (DSP Technology Inc., SigLab model 20-22) was used to measure the transfer function from actuator command to tooltip acceleration. While measuring the frequency response, we found that various factors influenced its response including the grip used to hold it. Therefore, we investigated the influence of the gripping method, of gripping force, and of the probed surface on device's response. In a first condition, see Figure 4a, the probe was resting flat on four extended fingers, thus causing minimal loading, and the acceleration of all three axes was measured. For the gripping-force factor, the experimenter held the probe tighter than normally, see Figure 4b. To study the effect of grip, the experimenter held the probe using two different methods: holding it as a pen, and holding it as surgical knife, see Figure 4c. To investigate the probed-surface factor, the experimenter touched different surfaces with the probe tip, see Figure $4 \mathrm{~d}$.
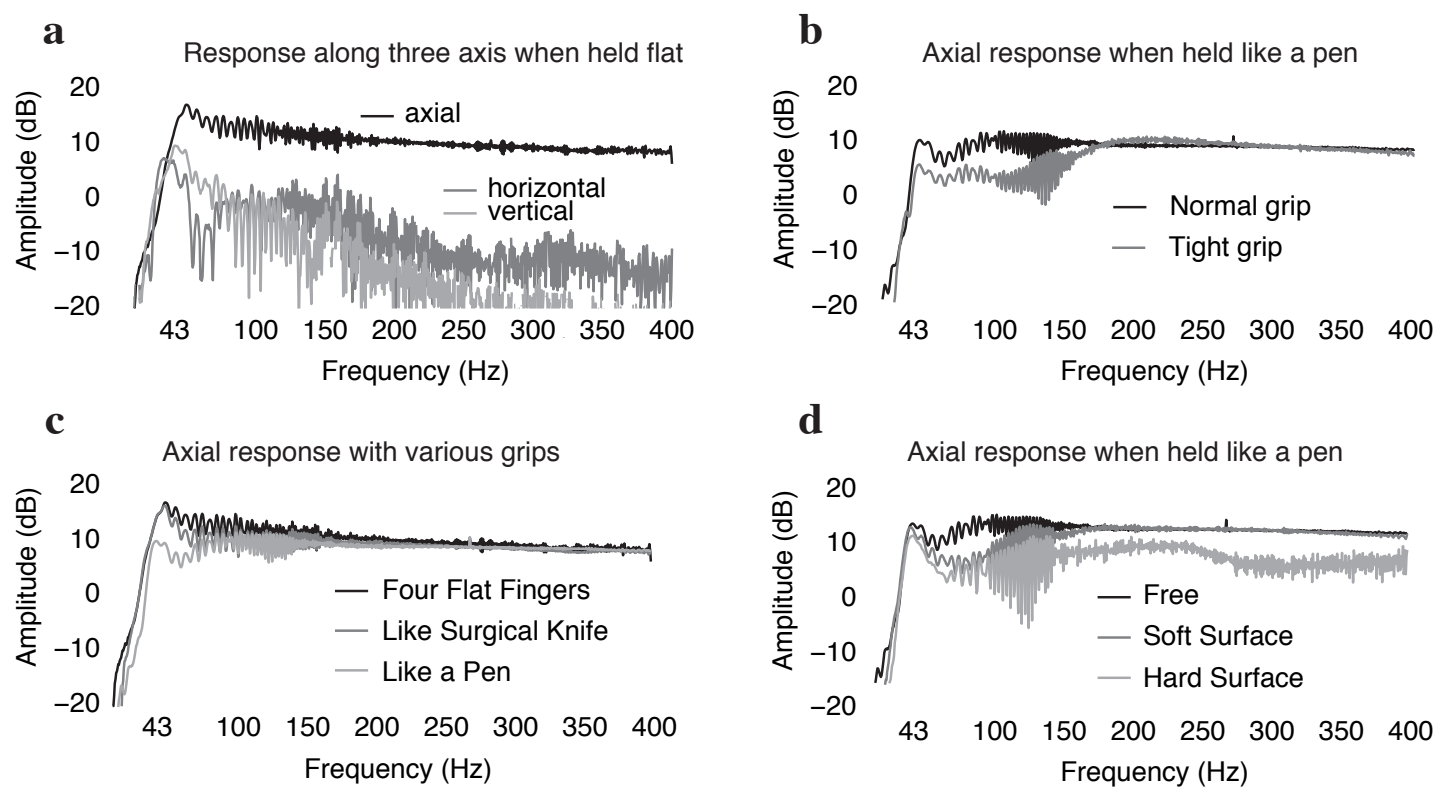

Figure 4: Responses grouped by holding method and probed surfaces.

\subsection{User Experiment Results}

The results are summarized by condition in Figure 5a and by pad in Figure 5b. The results show that subject performance improved with haptic and sound feedback. A significance test confirmed that the haptic and sound feedback both influenced the performance. Sound feedback improved performance by approximately $20 \%$, and haptic feedback by $10 \%$. One-way analysis of variance (ANOVA) of the three conditions Off, Haptic and Audio confirmed the significance of the differences $(p=0.015, p<0.05)$. The ANOVA test applied to pairs of conditions yielded $p<0.05$ for the Audio - Off, and Haptic - Off conditions. There was no significant difference between Haptic and Passive conditions 
$(p=0.15, p>0.05)$. The difference in performance between naive and non-naive subjects was not significant according to a 2 -way ANova test $(p=0.53, p>0.05)$. There was no significant difference between the physicians and the other subjects $(p>0.05)$.
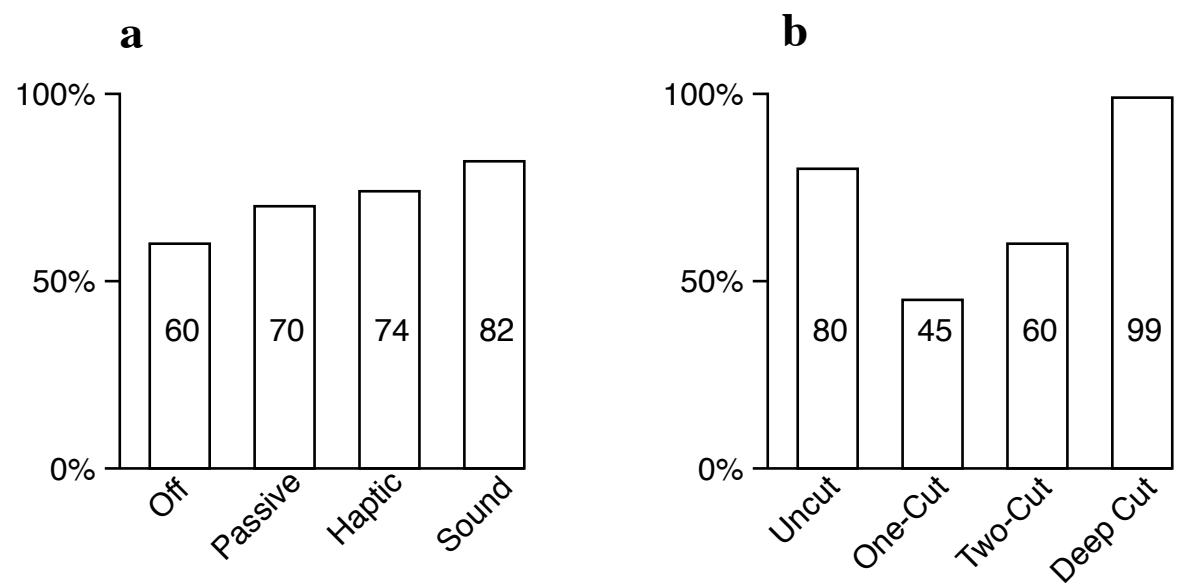

Figure 5: Results summarized (a) by condition, (b) by pad.

More detailed data are presented in Table 1. Deep cuts were almost perfectly detected, and most subjects also responded correctly for the surface with no cuts. For pads with small cuts, the performance in Haptic, Sound, and Passive was better than in the Off condition. When there was no feedback, the subjects failed to detect the presence of small cuts most of the time.

Table 1: Pooled results in percentile in the 4 conditions and for the different cuts.

\begin{tabular}{c|cccc} 
Condition & No cut & small cut & two cuts & deep cut \\
\hline Off & 92 & 15 & 33 & 100 \\
Haptic & 90 & 44 & 65 & 100 \\
Audio & 90 & 63 & 77 & 100 \\
Passive & 50 & 60 & 65 & 98
\end{tabular}

\section{Discussion}

\subsection{Device Characterization Discussion}

Figure 4 shows the frequency response under different conditions. The system gain was approximately $10 \mathrm{~dB}$ in all the cases, and higher gains would make the system unstable. This limitation was only valid for haptic reproduction; for auditory reproduction, the system gain was only limited by the capacity of the amplifier.

When the device was held flat on four extended fingers the response, as shown in Figure $4 \mathrm{a}$, was uniform in the axial direction and there was at least $10 \mathrm{~dB}$ of attenuation along the other directions (labeled 'horizontal' and 'vertical'). The grip type modified 


\section{Computer Aided Surgery, Vol. 10, No. 4, pp. 233-239.}

the response, as shown in Figure 4b. A tighter grip attenuated the response, as shown in Figure 4c. The response was also changed according to the hardness of the surface, with both attenuation in the low frequencies and excitation of resonances in the high frequencies for hard surfaces as seen in Figure 4d. These varying dynamic behaviors may be perceptually valuable to an experienced user because they provide rich information about the object being probed. However, further analysis of these effects according the type of feedback and the task being performed is needed to verify this conjecture.

\subsection{User Experiment Discussion}

For the uncut and the deeply cut pads, the performance was well above chance. The deep cut was almost perfectly detected under all conditions. The haptic and audio feedback did not have a negative influence on detection of a deep cut, and the subjects performed at least as well with feedback as without. Furthermore, in passive detection, the haptic signal was adequate for the correct detection of a deep cut. Without haptic or audio feedback, remote detection would of course be impossible and yet our subjects performed remarkably well: the $2 \%$ error rate for remote detection may well have been due to a single error made when the subject entered the data.

The results also showed that, with one or two small cuts, performance without enhanced feedback was not far from the rate predicted by chance. This suggested that the dimensions of the cuts were close to the threshold of detection. From the results in Table 1 we can conclude that, without feedback, the existence of a cut was hard to detect. With either auditory or haptic feedback, the detection rate increased. Thus the system was able to improve the performance of subjects in detecting superficial cuts in a cartilage-like material.

The tests of significance indicated that haptic and auditory feedback had positive influences on the performance. As for the passive cut-detection task, the performance was at least as good as with active exploration without augmentation. In the passive condition, subjects had no control of the probe and could not see how the experimenter explored the surface. When subjects used the probe actively, they could vary the speed and the pressure applied by the probe. However, subjects were still able to detect the cuts well, as shown in Table 1. For pads with small cuts, the performance in the Remote condition was similar to Off and Haptic conditions.

Performance with audio feedback was consistently better than with haptic feedback, and most subjects spontaneously contributed an opinion to this effect. The simplest explanation is that our auditory system is more able to detect small transients out of a noisy background than is our tactile system. It is also possible that, using the two combined modalities of touch and audition, sensitivity may increase. Another possible explanation is that some useful information was lost in the filtering process. The signals to the speakers were not processed, but for haptics the signals were filtered and downsampled in an attempt to attempt to eliminate sensor noise. Even though the $400 \mathrm{~Hz}$ threshold was imposed during the filtering, there may be some useful information above this frequency. Signal enhancement techniques beyond plain magnification in a frequency band may be useful. 


\section{Conclusions}

We have introduced the first example of a family of instruments designed to enhance touch while probing a surface. Measurements of its frequency response demonstrated a generally uniform frequency response under undisturbed conditions, but factors such as holding method and probing surface may alter this response. Our preliminary study found that significant task improvement happened when either haptic or auditory feedback were presented. This experiment showed that the disturbance factors had small perceptual effects and that the users could use this device with minimal training. The device also made it possible to experience a surface remotely. The device can be used as a texturerecording/play instrument, which has considerable potential for use in surgical simulation and training. The device might also be useful for other judgment and detection tasks.

This project is still at an early stage and many improvements are possible building on the basic principle described here. The device's structural dynamics might also be further studied and improved, and a toolbox of signal processing algorithms can be developed to enhance the performance of specific tasks.

\section{Acknowledgments}

This research was supported in part by the Institute for Robotics and Intelligent Systems, the Ontario Research and Development Challenge Fund, and the Natural Sciences and Engineering Research Council of Canada.

\section{References}

1. Kontarinis DA, Howe RD. Tactile Display of Vibratory Information in Teleoperation and Virtual Environments. Presence. 1995;4(4):387-402.

2. Okamura AM, Cutkosky MR, Dennerlein JT. Reality-Based Models for Vibration Feedback in Virtual Environments. IEEE/ASME T. on Mechatronics 2001;6(3):245-252.

3. Rosen J, Hannaford B, MacFarlane MP, Sinanan MN. Force controlled and teleoperated endoscopic grasper for minimally invasive surgery-experimental performance evaluation. IEEE T. on Biomedical Engineering. 2003;46(10):1212-1221.

4. Pai K, Rizun PR. The WHaT: A Wireless Haptic Texture Sensor. Proc. Eleventh Symposium on Haptic Interfaces for Virtual Environment and Teleoperator Systems. 2003

5. Dario P, Hannaford B, Menciassi A. Smart Surgical Tools and Augmenting Devices. IEEE T. on Robotics and Automation, 2003;19(5):782-792. 